Objectives: We aimed to evaluate the drug retention rates, causes of discontinuation and outcome after cessation of IFX.

Methods: We reviewed the charts of 850 patients with BS who were registered in our clinic between 2009 and 2013 and identified those who had used IFX. The charts of these patients were surveyed for demographic features, the reasons for IFX use, previous and concomitant drugs, IFX duration, reasons for discontinuation and time to flare after discontinuation of IFX. We defined flare as disease activity in the organ involvement that necessitated IFX use. New major organ involvement that developed during or after discontinuation of IFX were also be noted.

Results: A total of 50/850 patients were treated with IFX (40 men, mean age $40 \pm 9.5$ years), for uveitis $(n=29)$, vascular involvement $(n=11)$, parenchymal neurologic involvement $(n=8)$, arthritis $(n=1)$ and venous ulcer $(n=1)$. Of these 50 patients, 22 (43\%) are still receiving IFX for a median duration of 40 (IQR: 25-83) months. The remaining $28(47 \%)$ patients had discontinued IFX after a median follow-up of 12 (IQR: 7-30) months. Reasons for discontinuation were remission in 7 patients, adverse events in 10, primary lack of efficacy in 2, and lack of patient compliance in 9 patients. Among the 7 patients who discontinued IFX due to remission, only 1 patient with uveitis had a flare, 11 months after discontinuation, while on azathioprine. The remaining 6 did not experience any flares during a median follow-up of 29.5 (IQR: 4-24) months. Five of these patients used azathioprine and 1 used mycophenolate mofetil for maintenance. Among the 10 patients who discontinued due to adverse events, IFX was switched to adalimumab in 3 patients and none experienced flares under adalimumab. The remaining 7 patients continued to receive azathioprine or mycophenolate mofetil without a biologic. Among these, 1 patient with uveitis 1 with arthritis experienced flares 6 months after discontinuing IFX. Among the 9 patients who discontinued IFX due to lack of patient compliance, 3 patients ( 2 with uveitis and 1 with arthritis) had flares after 5 months, 1 year and 1.5 years. IFX was re-initiated in all. The remaining 6 patients did not experience any flares after a mean follow up of $5 \pm 1.5$ years. Two with uveitis and 2 with venous thrombosis used azathioprine for maintenance, while 2 patients did not receive further treatment. New major organ involvement was not observed. New BS manifestations developed in 2 patients under IFX, arthritis in one patient and both epididymitis and erythema nodosum in the other.

Conclusion: Almost half of our patients with BS remained on IFX during a median follow-up of 5.4 years (IQR:2.4-7). Main reasons for discontinuation were adverse events, remission and lack of patient compliance. Our observations further support the efficiency of IFX in managing patients with BS.

Disclosure of Interests: Sinem Nihal Esatoglu: None declared, Beyza Tukek: None declared, Sıtkı Safa Taflan: None declared, Yilmaz Ozyazgan: None declared, Didar Ucar: None declared, Emire Seyahi: None declared, Melike Melikoglu: None declared, Vedat Hamuryudan Speakers bureau: Pfizer, AbbVie, Amgen, MSD, Novartis, UCB, Ugur Uygunoglu: None declared, Aksel Siva: None declared, Izzet Fresko: None declared, Sebahattin Yurdakul: None declared, Hasan Yazici: None declared, Gulen Hatemi Grant/research support from: BMS, Celgene Corporation, Silk Road Therapeutics - grant/research support, Consultant of: Bayer, Eli Lilly - consultant, Speakers bureau: AbbVie, Mustafa Nevzat, Novartis, UCB - speaker

DOI: 10.1136/annrheumdis-2020-eular.3299

\section{SAT0259 ANCA-ASSOCIATED VASCULITIS WITH RENAL INVOLVEMENT: THE ROLE OF A COMBINED HISTOPATHOLOGICAL ASSESSMENT AS PREDICTOR} OF PATIENTS' PROGNOSIS

L. Gigante ${ }^{1}$, P. G. Cerasuolo ${ }^{1}$, G. Vischini ${ }^{2}$, F. Federico ${ }^{3}$, D. Bruno ${ }^{1}$, A. Musto ${ }^{1}$, S. Costanzi ${ }^{2}$, S. L. Bosello ${ }^{1,4}$, E. Gremese ${ }^{1,4} .{ }^{1}$ Division of Rheumatology, Università Cattolica del Sacro Cuore, Rome, Italy; ${ }^{2}$ Division of Nephrology Fondazione Policlinico Universitario A. Gemelli-IRCCS, Rome, Italy; ${ }^{3}$ Division of Anatomical Pathology, Università Cattolica del Sacro Cuore, Rome, Italy: ${ }^{4}$ Division of Rheumatology, Fondazione Policlinico Universitario A. Gemelli, IRCCS, Rome, Italy

Background: Anti-neutrophil cytoplasmic antibody (ANCA)-associated vasculitis often affect the kidney and renal involvement has a considerable clinical impact on patient's prognosis. Currently used histopathological classifications are basically focused on the glomerular damage and assessing chronic damage progression, but their prognostic role presented some limitations.

Objectives: To combine the Berden Classification, the ANCA Renal Risk Score (ARRS) and the Mayo Clinic-Renal Chronicity Score (RCS) with the inflammatory interstitial infiltrate and to evaluate the prognostic value of the combined assessment in patients with AAV

Methods: We included 19 AAV patients with renal involvement (mean age $63 \pm 13.2$ years; disease duration $4.9 \pm 5.2$ months) who underwent renal biopsy. Patients were classified according to age, sex, disease duration, ANCA positivity. The histopathological evaluation was performed assessing the Berden category, Risk group (low, medium, high) according to the ARRS and Chronicity class according to the RCS; we also assessed the \% of inflammatory interstitial infiltrate. Each patient was followed-up for 12 months; we considered the stage IV (eGFR $\left.<30 \mathrm{ml} / \mathrm{min} / \mathrm{m}^{2}\right)$ of the KDIGO CKD Classification as renal outcome.

Results: 8 (42.1\%) AAV patients were p-ANCA and 11 (57.9\%) c-ANCA. 12 months after renal biopsy, 8 patients $(42.1 \%)$ had a GFR $<30 \mathrm{ml} / \mathrm{min}$. According to the ARRS, $10(52.6 \%)$ patients were in low, $7(36.8 \%)$ in medium and $2(10.5 \%)$ in high risk group. According to the RCS, $2(10.5 \%)$ biopsies had minimal, $10(52.6 \%)$ mild and $7(36.8 \%)$ moderate chronic changes, no one presented severe chronic changes. According to the Berden classification, $6(31.6 \%)$ samples represented the focal, $2(10.5 \%)$ the crescentic and $11(57.9 \%)$ the mixed category, no one represented the sclerotic class. The mean $\%$ of inflammatory infiltrate was $37.4 \pm 25.2$. The interstitial inflammatory infiltrate showed a direct correlation with the severity of the Berden category $(R=0.51 ; p=0.025)$, the \% of sclerotic glomeruli $(R=0.6 ; p=0.007)$ and the number of fibrocellular crescents $(0.46 ; p=0.05)$ and an inverse correlation with the GFR at 12 months $(R=-0.48 ; p=0.045)$. A ROC curve study identified a $22.5 \%$ cut-off of inflammatory infiltrate to predict the outcome of GFR a 12 months $<30 \mathrm{ml} / \mathrm{min}$ (sensitivity $88 \%$, specificity $97.5 \%$ ). Patients in focal class developed less frequently a GFR $<30(x 2=9.1 ; p=0.003)$, but there were no differences in the outcomes between the crescentic and mixed class. ARRS could differentiate risk group with regard to the renal outcome stage IV $\left(x^{2}=9.0\right.$ e $\left.p=0.01\right)$ as well as the chronicity Score $\left(x^{2}=8.1 ; p=0.017\right)$. Finally, we built a matrix combining the different histopathological scores and the $\%$ of inflammatory infiltrate to predict the outcome; we found that an inflammatory infiltrate wider than $22.5 \%$ characterizes most of patients developing stage IV chronic renal failure at the 12th month. In fact, more than $75 \%$ of patients with eGFR $<30 \mathrm{ml} / \mathrm{min}$ had inflammatory infiltrate wider than $22.5 \%$ at biopsy, despite they were in the low risk class (ARRS) and in minimal changes class (RCS).

Conclusion: Our results underline the importance of the inflammatory infiltrate in renal outcome and histology. Despite the limited number of patients, our data suggest that a combined histological score assessing the chronicity and activity of renal disease from both glomerular and interstitial perspective could better predict patients' global and renal prognosis.

References:

[1] Berden, J Am Soc Nephrol, 2010 Berti, Nephrol Dial Transplant 2018 Brix, Kidney Int. 2018

Disclosure of Interests: Laura Gigante: None declared, Pier Giacomo Cerasuolo: None declared, Gisella Vischini: None declared, Francesco Federico: None declared, Dario Bruno: None declared, Alessia Musto: None declared, Stefano Costanzi: None declared, Silvia Laura Bosello Speakers bureau: Abbvie, Pfizer, Boehringer, Elisa Gremese Speakers bureau: Abbvie, BMS, Celgene, Jannsen, Lilly, MSD, Novartis, Pfizer, Sandoz, UCB DOI: 10.1136/annrheumdis-2020-eular.5676

\section{SAT0260 1 PENTOXYFILLINE GEL FOR ORAL ULCERS IN PATIENTS WITH BEHÇET'S SYNDROME}

G. Hatemi ${ }^{1}$, B. Yurttas ${ }^{1}$, Z. Kutlubay ${ }^{2}$, T. Cote $^{3}$, Ş. B. Derkunt ${ }^{3}$, Y. Yazici ${ }^{4}$, H. Yazici ${ }^{5}{ }^{1}$ Istanbul University - Cerrahpasa, Cerrahpasa Medical Faculty, Department of Internal Medicine, Division of Rheumatology, Behçet's Disease Research Center, Istanbul, Turkey; ${ }^{2}$ stanbul UniversityCerrahpasa, Cerrahpasa Medical Faculty, Department of Dermatology, Behçet's Disease Research Center, Istanbul, Turkey; ${ }^{3}$ Silk Road Therapeutics, Washington, D.C., United States of America; ${ }^{4}$ New York University School of Medicine, New York, NY, United States of America; ${ }^{5}$ Academic Hospital, Istanbul, Turkey

Background: Oral ulcers, the hallmark lesion of Behçet's syndrome (BS) can be disabling and impair eating, drinking and speaking. Despite recent advances in systemic medications for the treatment of oral ulcers, some patients do not achieve complete remission. Topical agents may help such patients by decreasing the pain and duration of oral ulcers. Pentoxyfilline (PTX) is a methylxanthine derivative that inhibits phosphodiesterase and is thought to have immunomodulatory effects in addition to improving blood flow which is its main reason for use in peripheral vascular disorders.

Objectives: The aim of this study is to assess the efficacy and safety of PTX gel for oral ulcers in patients with BS. We also aimed to explore the best tools for the assessment of treatment response to topical agents in randomized controlled trials (Clinicaltrial.gov ID: NCT 03888846). 
Methods: This was an open-label, randomized, parallel group study comparing PTX gel in addition to colchicine (PTX-COL) with colchicine alone (COL). Patients with BS who were treated with colchicine and not using any other systemic medications for BS, having at least one oral ulcer that appeared during the last 48 hours were included. PTX $5 \%$ gel with a dose of $1000 \mathrm{mg} /$ day was applied in 4 divided doses per day for 14 days. Patients were contacted daily for 14 consecutive days. Photographs were taken every 24 - 48 hours and graphical processing software was used to calculate the area of the index ulcer. Duration of the index ulcer, time to start of index ulcer shrinkage, time to $50 \%$ reduction in oral ulcer pain on a $10 \mathrm{~mm}$ visual analog scale (VAS), change from baseline in the area of the index ulcer over time, total number of oral ulcers and adverse events were evaluated.

Results: A total of 41 patients were randomized, 39 patients (18 in the PTX-COL group and 21 in the COL group) completed the study and 2 patients in PTX-COL group withdrew from the study due to unacceptable dysgeusia and nausea. Mean duration of index ulcer, time to start of index ulcer shrinkage, time to $50 \%$ reduction in oral ulcer pain, and number of patients with no detectable ulcers on day 4 in each group were lower in the PTX-COL group as presented in the Table. Change from baseline in the area of index ulcer and pain score over time is shown in the Figure. There were no serious adverse events. Fifteen (75\%) patients reported nausea, 11 (55\%) reported dysgeusia and 2 reported vomitting in the PTX-COL group, while 2 patients (10\%) reported nausea in the COL group.

Conclusion: This pilot phase 2 open label, randomized controlled study supports the hypothesis that topical PTX in addition to colchicine accelerates the healing of BS oral ulcers compared to colchicine alone. A phase 3 controlled study with a higher number of patients is planned with improving the taste for tolerability of the product.

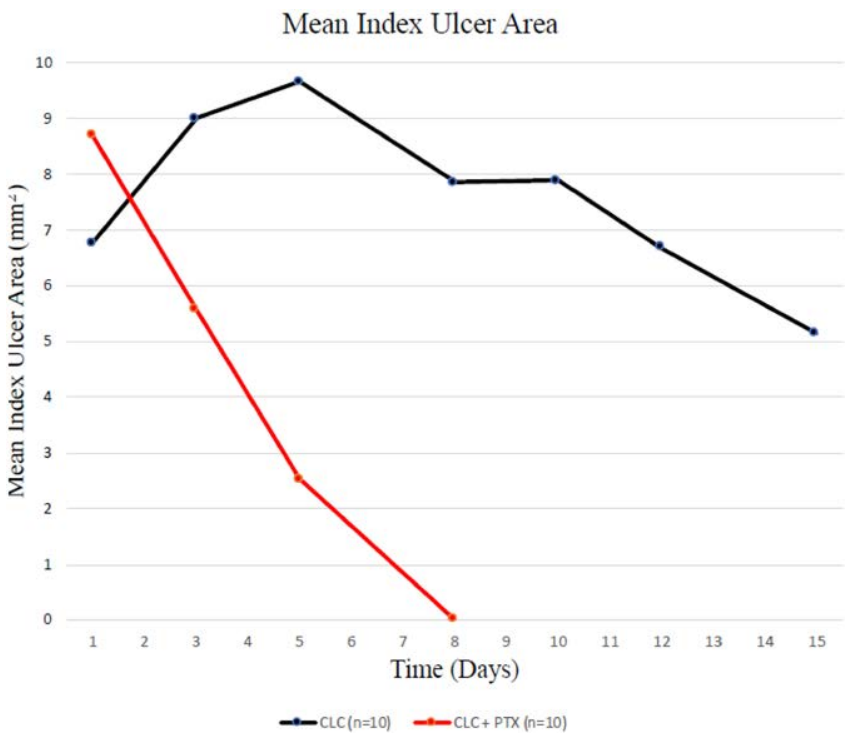

Disclosure of Interests: Gulen Hatemi Grant/research support from: BMS, Celgene Corporation, Silk Road Therapeutics - grant/research support, Consultant of: Bayer, Eli Lilly - consultant, Speakers bureau: AbbVie, Mustafa Nevzat, Novartis, UCB - speaker, Berna Yurttas: None declared, Zekayi Kutlubay: None declared, Tim Cote Employee of: Silk Road Therapeutics is in Washington, DC, USA, Şemsi Burak Derkunt Employee of: Silk Road Therapeutics is in Washington, DC, USA, Yusuf Yazici: None declared, Hasan Yazici: None declared

DOI: 10.1136/annrheumdis-2020-eular.5610

\section{SAT0261 FEATURES AND RISK FACTORS OF SERIOUS INFECTIONS IN ANCA ASSOCIATED VASCULITIS: LONG TERM FOLLOW UP OF 186 PATIENTS}

B. Ince ${ }^{1}$, M. Bektas ${ }^{1}$, F. Kemik ${ }^{2}$, A. E. Aytun ${ }^{3}$, Y. Yalçınkaya ${ }^{1}$, B. Artim-Esen¹, A. Gül ${ }^{1}$, M. L. Ocal ${ }^{1}$, M. Inanc ${ }^{1} .{ }^{1}$ Istanbul University, Istanbul Faculty of Medicine, Dept. of Internal Medicine, Division of Rheumatology, Istanbul, Turkey; ${ }^{2}$ Istanbul University, Istanbul Faculty of Medicine, Dept. of Internal Medicine, Istanbul, Turkey; ${ }^{3}$ Istanbul Faculty of Medicine, Istanbul, Turkey

Background: Serious infections (SI) are one of the main complications in patients with ANCA associated vasculitis (AAV).

Objectives: We planned to investigate the prevalence, features and risk factors of $\mathrm{SI}$ in our AAV cohort during follow-up.
Methods: Outpatient and hospital data of patients diagnosed with granulomatous polyangiitis (GPA), microscopic polyangiitis (MPA) and eosinophilic granulomatous polyangiitis (eGPA) between 1999 and 2019 according to Chapel Hill Consensus Criteria and followed up at least 6 months in our vasculitis clinic were evaluated. Development of sepsis, requirement for intravenous (IV) antibiotherapy and / or hospitalization during infection episodes were considered as SI. Chisquare, student's t-test and logistic regression analysis were used for statistical analysis.

Results: Study was conducted with 186 (53.6\% female) patients with adequate follow-up data. Mean age of diagnosis was 54.3 $\pm 14,5$ (23-79), mean follow-up duration was $86,4 \pm 54,3(6-251)$ months. Number of GPA, MPA and eGPA patients were $132(71 \%), 42(22,5 \%)$ and $12(6,5 \%)$, respectively. IV cyclophosphamide (CYC) was used in $148(79,6 \%)$, azathioprin in $105(56,5 \%)$, rituximab (RTX) in 69 (\%37,1), methotrexate in $29(15,6 \%)$ and mycophenolate mofetil in 14 (7,5\%) patients. Number of patients developed SI was 66 (34.7\%), total SI episode was 86 , patients who had multiple episodes was 15. All SI is shown in Table-1. Bacterial pneumonia was the most common diagnosis and 26 of $\mathrm{SI}(30.2 \%)$ were considered as opportunistic (systemic viral, parasite, fungus) infections. Thirty-one of patients developed $\mathrm{SI}(40,7 \%)$ in the first year after diagnosis. SI were observed more frequently in the presence of major organ involvement (kidney, lung, neurological) $(65 / 173$ vs. $1 / 13 \mathrm{p}=0.02 \mathrm{OR}=8.795 \% \mathrm{Cl} 1.06-64.4)$. Diffuse alveola hemorrhage (DAH) was associated with $\mathrm{SI}$ in multivariate analysis (12/52 vs. 0/34 $\mathrm{p}=0.007 \mathrm{OR}=1.695 \% \mathrm{Cl} 1.3-1.96)$. Cumulative CYC dose was significantly highe in patients with $\mathrm{SI}(14,2 \pm 21$ vs. $8.2 \pm 13.9 \mathrm{p}=0.045)$. During maintenance, patients treated with RTX had significantly more $\mathrm{SI}$ (18/53 vs. $17 / 99 \mathrm{p}=0.19 \mathrm{OR}=3,395 \%$ $\mathrm{Cl} 1,55-7,07)$. Hypogammaglobulinemia (HIgG) $(\mathrm{lgG}<700 \mathrm{mg} / \mathrm{dL})$ was present in $12(14 \%)$ SI episodes. HIgG was associated with SI in RTX-treated patients (5/13 vs. $7 / 47 \mathrm{p}=0.03 \mathrm{OR}=4.2, \mathrm{Cl}=1-16.5)$. Hospitalization need for $\mathrm{SI}$ was $65 \%$. Disease flares (34/128 vs. $32 / 62 \mathrm{p}=0.001 \% 95 \mathrm{Cl}=2.995 \% \mathrm{Cl} 1.6-5.6)$ and organ damage presence were more common (64/65 vs. $109 / 125 p=0.0195 \%$ OR $=8.9$ $95 \% \mathrm{Cl} 1.1-68.9)$ in patients with a history of $\mathrm{SI}$ in multivariate analysis. SI was confirmed as cause of death in three cases.

Conclusion: Long-term follow-up results of a single center cohort of AAV patients revealed that approximately one third of patients developed SI, most frequently in the first year of treatment. During the maintenance period, the risk of SI continues. Cumulative CYC dosage and maintanence with RTX is associated with $\mathrm{SI}$, especially in patients who developed hlgG. Major organ involvement, disease flares and organ damage are significant risk factors for SI. In this regard, protection measures (vaccination, prophylaxis) should be reviewed and the quality of follow-up should be improved.

Table 1. Serious infections in AAV patients.

\begin{tabular}{|c|c|c|c|c|c|c|}
\hline BACTERIAL & $\mathrm{N}$ & FUNGAL & $\mathrm{N}$ & VIRAL & $\mathrm{N}$ & PROTOZOAN \\
\hline Pneumonia & 37 & PJP & 7 & Zoster Zona & 3 & $\begin{array}{c}\text { Intramuscular abscess } \\
\text { (Nocardiosis) }\end{array}$ \\
\hline $\begin{array}{l}\text { Urinary Tract } \\
\text { Infection (UTI) }\end{array}$ & 8 & Aspergilloma & 1 & CMV Pneumonia & 1 & \\
\hline $\operatorname{Gr}(-)$ sepsis & 2 & $\begin{array}{l}\text { Invasive Fungal } \\
\text { Infection }\end{array}$ & 3 & CMV Colitis & 1 & \\
\hline Perianal abscess & 2 & $\begin{array}{l}\text { Candida } \\
\text { Eosephagitis }\end{array}$ & 4 & CMV Gastritis & 1 & \\
\hline $\begin{array}{l}\text { Intraabdominal } \\
\text { abscess }\end{array}$ & 2 & Candidemia & 2 & $\begin{array}{c}\text { HSV } \\
\text { Eosephagitis }\end{array}$ & 1 & \\
\hline Catheter infection & 2 & UTI & 1 & & & \\
\hline Sellulitis & 1 & Fungal otitis & 1 & & & \\
\hline Orbital sellulitis & 1 & & & & & \\
\hline $\begin{array}{l}\text { Maxillary sinüs } \\
\text { abscess }\end{array}$ & 1 & & & & & \\
\hline Mastoiditis & 1 & & & & & \\
\hline $\begin{array}{l}\text { Prosthesis } \\
\text { infection }\end{array}$ & 1 & & & & & \\
\hline Septic artrhitis & 1 & & & & & \\
\hline Lung tuberculosis & 1 & & & & & \\
\hline
\end{tabular}

Disclosure of Interests: None declared

DOI: 10.1136/annrheumdis-2020-eular.4397

\section{\begin{tabular}{|l|l}
\hline SAT0262 & PROPOSAL FOR OPTIMIZATION OF DIAGNOSTIC
\end{tabular} IMAGING FOR GIANT CELL ARTERITIS USING THREE-DIMENSIONAL COMPUTED TOMOGRAPHY ANGIOGRAPHY IMAGE AND CONSTRUCTING VASCULAR MAPPING FROM VASCULAR ULTRASONOGRAPHY AS REFERENCES}

T. Kawamoto ${ }^{1}$, M. Ogasawara ${ }^{1}$, Y. Mastuki-Muramoto ${ }^{1}$, T. Kawaguchi $^{1}$, S. Ando ${ }^{1}$, M. Matsushita ${ }^{1}$, K. Yamanaka ${ }^{2}$, K. Yamaji ${ }^{1}$, N. Tamura ${ }^{1} .{ }^{1}$ Juntendo University 